Revista em Agronegócio e Meio Ambiente, Maringá (PR)

DOI: http://dx.doi.org/10.17765/2176-9168.2017v10n2p533-553

\title{
VARIABILIDADE ESPACO-TEMPORAL DE PARÂMETROS DE QUALIDADE DA ÂGUA AO LONGO DE SEÇÃO DE MONITORAMENTO DO RIO IGUAÇU
}

\author{
Vanessa Daneluz Gonçalves* \\ Cristovão Vicente Scapulatempo Fernandes**
}

RESUMO: As NBRs no 9.897 (ABNT, 1987a) e $\mathrm{n}^{0} 9.898$ (ABNT, 1987b) auxiliam no monitoramento de recursos hídricos de modo a sugerir a localização e o número de postos amostrais, inclusive aqueles ao longo de uma seção transversal do rio que variam conforme a sua largura e profundidade. Contudo, diante da complexidade e dos recursos necessários à prática de monitoramento de rios, é comum sacrificar tal amostragem para fazê-la de uma margem do rio. Para verificar o grau de influência do local de amostragem, avaliou-se neste trabalho a variação de concentrações de parâmetros de qualidade da água ao longo de uma seção transversal de rio, em diferentes condições hidrológicas. Para tanto, foram monitorados cinco pontos amostrais distribuídos ao longo da seção de estudo. Concluiu-se que houve elevada dispersão ao longo da seção de rio quanto aos parâmetros orgânicos, e uma tendência particular de distribuição gradual da concentração para cada parâmetro de DBO e DQO. Contudo, a Análise de Variância permite afirmar que não houve diferenças significativas entre os pontos amostrais. Com base no exposto, não houve impactos negativos à gestão de recursos hídricos decorrentes de informações de qualidade da água amostradas às margens do rio. Assim, para este estudo de caso, o número de pontos amostrais sugerido pela norma supracitada pode ser simplificado em um único ponto na seção de monitoramento.

PALAVRAS-CHAVE: Análise de variância; DBO; Dispersão amostral.

\section{SPACE-TIME VARIABILITY OF WATER QUALITY PARAMETERS THROUGHOUT THE MONITORED SEGMENT OF THE RIVER IGUAÇU}

ABSTRACT: NBRs n. 9.897 (ABNT, 1987a) and n. 9.898 (ABNT, 1987b) are a help

\footnotetext{
Mestre em Engenharia de Recursos Hídricos e Ambiental; Universidade Estadual de Maringá (UEM), Departamento de Tecnologia, Área de Engenharia Ambiental e Tecnologia em Meio Ambiente, Brasil, E-mail: van_dg@msn.com

** Doutorado em Civil and Environmental Engineering; Universidade Federal do Paraná, Departamento de Hidráulica e Saneamento, Área de Engenharia de Recursos Hídricos e Ambiental, Brasil.
} 
in the monitoring of hydric resources since they suggest the site and number of sample sites, including those throughout the transversal section of the river which vary according to breadth and depth. In the wake of the complexity and resources required for the monitoring of rivers, usually such sampling is discarded and replaced by the river's margin. So that the sampling site's influence may be verified, the variations of the concentrations of water quality parameters throughout the river's transversal section are evaluated at different hydrological conditions. Five sampling sites distributed along the studied segment were monitored. There was a great dispersion throughout the river's segment with regard to organic parameters and there was a specific trend in gradual distribution of concentration for each BOD and COD parameter. The analysis of variance showed that there was no significant difference between sampling sites. There were no negative impacts with regard to the management of hydric resources caused by information on water quality samples at the river's margin. In current study case, the number of sites suggested by the above norm may be simplified to a single site within monitoring.

KEY WORDS: Analysis of variance; DBO; Sample dispersion.

\section{INTRODUÇÃO}

O monitoramento de parâmetros de qualidade da água previstos na resolução CONAMA n ${ }^{0}$ 357, de 2005 (BRASIL, 2005), surge como uma ferramenta fundamental para avaliação da qualidade do corpo hídrico, com vistas à sustentabilidade da biota aquática, além de subsidiar um histórico evolutivo do ambiente hídrico e corroborar com a definição de metas progressivas de despoluição hídrica quando for o caso (GONÇALVES, 2011). O monitoramento de recursos hídricos deve estar em conformidade com certas exigências metodológicas, que são por vezes negligenciadas, quanto às atividades de coleta, armazenamento e análise de amostras durante a execução de ensaios bioquímicos ou físicos (APHA, 1998; ABNT, 1992). Entre os ensaios bioquímicos, o de demanda bioquímica de oxigênio - DBO, de acordo com Kamiyama (1988), pode ser destacado pelo seu elevado nível de complexidade analítica. Para o autor a experimentação da DBO é realizada de modo indiscriminado e exacerbado, e em muitos casos com o agravante da sua compreensão e/ou execução de maneira deficitária ou até mesmo errônea.

Para minimizar possíveis erros na aquisição de informações e dados de 
qualidade, por exemplo, é comum a adoção de normas e padronizações como as Normas Brasileiras - NBRs no 9.897 (ABNT, 1987a) e $\mathrm{n}^{\circ} 9.898$ (ABNT, 1987b), as quais fornecem suporte técnico e organizacional para as atividades de monitoramento da qualidade da água, ao prescreverem respectivamente quanto ao Planejamento de Amostragem de Efluentes Líquidos e Corpos Receptores; e quanto à Preservação e Técnicas de Amostragem de Efluentes Líquidos e Corpos Receptores. Entre as sugestões dispostas nas normativas, no que tange à amostragem de recursos hídricos, para rios pequenos, a coleta de amostras em um único ponto na seção transversal do rio contém orientações para ser efetuada no meio da seção transversal e a meia profundidade. Entretanto, em rios extensos transversalmente e/ou profundos, a norma sugere um maior número de pontos amostrais em profundidade e/ou ao longo da seção transversal do rio em estudo (ABNT, 1987a). Esta prática apresenta maior grau de complexidade, tanto pela necessidade de um maior número de pontos de monitoramento na seção, quanto pela necessidade de equipamentos mais sofisticados como um barco a motor juntamente com operador capacitado, por exemplo. Somada ao supracitado, há a demanda por recursos financeiros, geralmente indisponíveis ou disponíveis de forma burocrática.

Efetuar um monitoramento em conformidade com as NBRs citadas podese tornar dispendioso e são raramente mencionados na literatura. A exigência quanto ao número e localização dos pontos amostrais pode ser sustentada por conceitos de fluxo de velocidades, e consequentemente por fenômenos de advecção e difusão, em que se pressupõe que a dinâmica de fluxo de rios pode assumir um caráter de mistura completa ou possuir zonas mortas. Contudo, o predomínio de fenômenos de advecção ou difusão, além dos relevantes aspectos de comprimento ou zona de mistura, ao longo de rio são dependentes de características peculiares do mesmo, como a morfologia, sinuosidade, declividade, velocidade do escoamento, etc. Distintos trabalhos versam sobre a variabilidade sazonal de parâmetros de qualidade de corpos hídricos ou mesmo sobre a variabilidade especial ao longo de um rio (CAMPANHA et al., 2010; COELHO; KISHI, 2011; SANTI et al., 2012; BARBOT et al., 2013), ou ainda quanto a uma otimização da localização amostral ao longo do rio (MEMARZADEH et al., 2013) e da frequência do monitoramento (LIU et al., 2013). Contudo, pouco se discute sobre a variabilidade espacial ao longo de uma seção 
transversal de rio. Em referências bibliográficas em temas de gestão de qualidade da água, aspectos de confirmação de zona de mistura são sequer questionados (SANDERS et al., 1983; STROBL et al., 2008).

Estes pressupostos são bastante conceituais, sendo precárias as informações quanto aos fenômenos existentes na coluna d'água de rios pequenos ou grandes, e sua interação com leito de fundo, como estudo desenvolvido por Reis et al. (2001) sobre o sedimento. A inexistência de estudos no Brasil com esta preocupação e a falta de trabalhos ilustrativos deste tipo de questionamento resultam na falta de investigação quanto aos fenômenos que ocorrem na coluna d'água e, consequentemente, a real variação dos parâmetros de qualidade da água ao longo de uma seção transversal de rio (GONÇALVES, 2011).

Desta forma, a presente pesquisa propõe investigar os fenômenos existentes ao longo de uma seção transversal de rio, e concomitantemente, avaliar a necessidade de adoção do sugerido pelas NBRs mencionadas quanto à estipulação de localização e número de pontos amostrais em atividades de monitoramento. Para implantação do monitoramento quali-quantitativo, utilizou-se como estudo de caso uma seção transversal do rio Iguaçu, situado na Bacia do Alto Iguaçu, na Região Metropolitana de Curitiba. Almeja-se, com este trabalho, consolidar informações e conhecimentos científicos consistentes, para subsidiar o planejamento e a gestão de recursos hídricos.

\section{MATERIAL E MÉTODOS}

\section{1 ÁREA DE ESTUDO - SEÇÃO TRANSVERSAL DE RIO}

O monitoramento foi realizado no rio Iguaçu enquadrado na classe 2 pela Portaria SUREHMA no 20/1992 (PARANÁ, 1992), e pertencente à Bacia do Alto Iguaçu, a qual drena cerca de $3.000 \mathrm{~km}^{2}$ de área. A bacia está situada no Estado do Paraná e engloba total ou parcialmente a cidade de Curitiba, e mais outros 14 municípios denominados por Região Metropolitana de Curitiba - RMC, o que inclui o município de Araucária. O trecho do rio Iguaçu, pertencente à Bacia do Alto Iguaçu, possui $86 \mathrm{~km}$ de extensão, desde a sua nascente na junção dos rios Iraí e Palmital, 
até a sua foz no rio Verde, incluindo 26 afluentes principais ao rio Iguaçu (PORTO et al., 2007; KNAPIK, 2009). Os afluentes de margem direita cortam a cidade de Curitiba e recebem toda a carga proveniente de poluição difusa, efluente doméstico, lançamentos pontuais e efluentes resultantes das indústrias instaladas na Cidade Industrial de Curitiba - CIC, situada no terço inferior da sub-bacia do rio Barigui, afluente ao rio Iguaçu (FRANÇA, 2009).

Para a avaliação da variabilidade espacial ao longo da seção transversal do rio definiu-se como local de estudo a seção transversal do rio Iguaçu situada no município de Araucária, sob as coordenadas $25^{\circ} 36^{\prime} 01^{\prime \prime}$ Sul e $49^{\circ} 30^{\prime} 48^{\prime \prime}$ Oeste. Distintos estudos de monitoramento da qualidade da água foram desenvolvidos neste local (PORTO et al., 2007; KNAPIK, 2009; FRANÇA, 2009), contudo com uma amostragem pontual na seção, diferente do proposto por este trabalho que busca identificar a variabilidade espaço-temporal ao longo da seção de rio. Neste contexto, a seção possui cerca de 80 metros de largura e profundidade variável com a vazão do rio, a qual assume o valor de 4,10 metros em uma condição de $243,80 \mathrm{~m}^{3} \mathrm{~s}^{-1}$ de vazão no canal. A seção está a uma altitude de 858 metros, e sob influência de uma área de drenagem que compreende cerca de $2.577,76 \mathrm{~km}^{2}$ (PORTO et al., 2007; KNAPIK, 2009). A Figura 1 apresenta a localização da seção transversal do rio Iguaçu em estudo, no âmbito da Bacia do Alto Iguaçu.

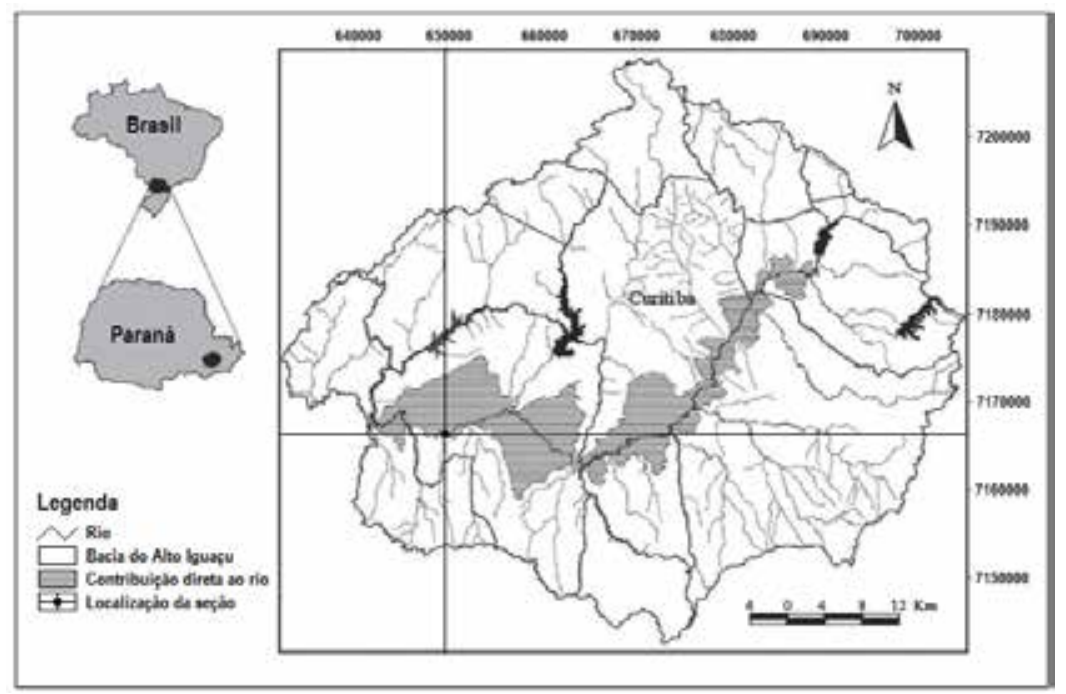

Figura 1. Localização da seção transversal de rio de monitoramento Fonte: Adaptado de Silva et al., 2010. 


\subsection{LOCALIZAÇÃO DA AMOSTRAGEM}

Em uma visita de inspeção ao local de estudo foram registrados os parâmetros médios de profundidade e largura de $48 \mathrm{~m} \mathrm{e} 2 \mathrm{~m}$, respectivamente. Logo, com base na normativa NBR 9.897 (ABNT, 1987a), ao longo da seção transversal do rio em estudo foram estipulados da direita para a esquerda, cinco pontos amostrais P1, P2, P3, P4 e P5. Sendo, P1 e P5, de margem direita e esquerda respectivamente, e

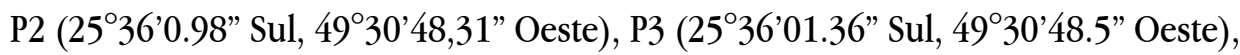

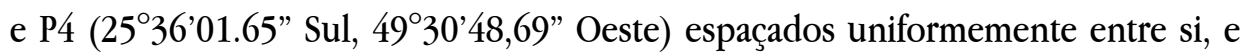
a meia profundidade como ilustrado na Figura 2. Salienta-se que as dimensões de espaçamento e profundidade supracitadas foram variáveis de acordo com a condição de vazão do rio registrada no momento da amostragem. As coletas foram realizadas no período de novembro de 2009 e setembro de 2010, no qual registrou-se uma variação de vazão de $27,30 \mathrm{~m}^{3} \cdot \mathrm{s}^{-1}$ a $102,44 \mathrm{~m}^{3} \cdot \mathrm{s}^{1}$.

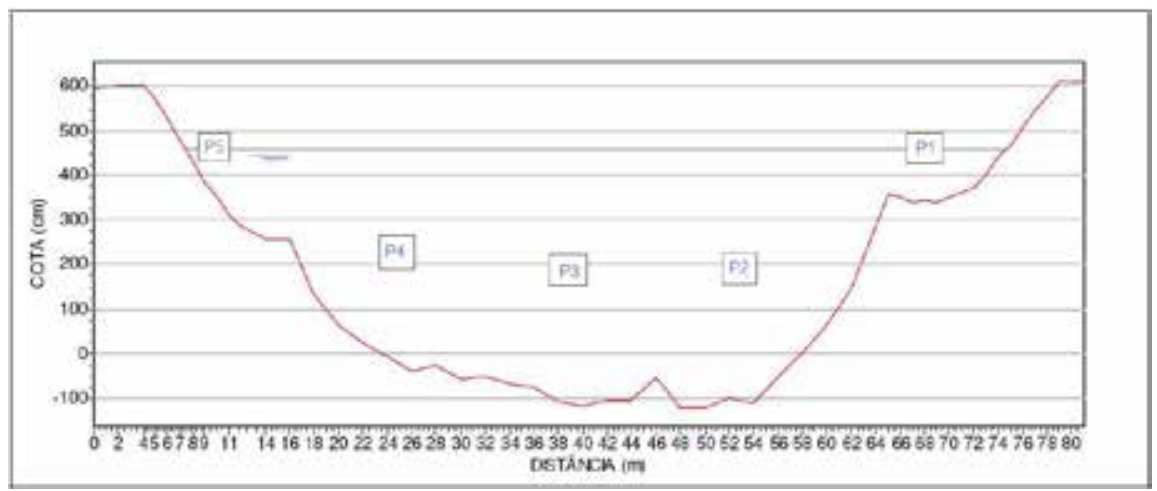

Figura 2. Pontos de Monitoramento.

Fonte: Adaptado de Porto et al. (2007).

As amostras foram coletadas a meia profundidade com auxílio de uma garrafa de Van Dorn içada simultaneamente com um guincho elétrico detentor das informações de profundidade do local. Na sequência, as amostras foram reservadas em frascos de poliestireno e mantidas sob refrigeração com auxílio de caixa térmica e gelo, como sugerido na NBR 9.898 (ABNT, 1987b), até a sua avaliação em termos de qualidade em laboratório específico de análises ambientais, sempre respeitando 
os prazos de validade de cada análise conforme sugerido em APHA (1998) e ABNT (1992). Parâmetros mensuráveis por sensores específicos de campo foram determinados in loco.

\subsection{PARÂMETROS DE QUALIDADE DA ÁGUA MONITORADOS}

Foram realizadas seis coletas amostrais com a determinação dos seguintes parâmetros de qualidade da água: condutividade (sensor Handylab LF1, SCHOTT); pH - potencial hidrogeniônico (pH 330i/SET, WTW); turbidez (WQ770 Turbidimeter, Global Water); oxigênio dissolvido - OD (Handylab OX 12/SET, SCHOTT); temperatura (obtido pelo Oxímetro); Carbono Orgânico Dissolvido CODT ou COD (Combustão e detecção por infravermelho não dispersivo - NDIR, TOC-VCPH SHIMADZU CORPORATION, 2003); Demanda Bioquímica de Oxigênio - DBO; e Demanda Química de Oxigênio - DQO (APHA, 1998).

Neste contexto, os parâmetros de condutividade, $\mathrm{pH}$, turbidez, oxigênio dissolvido e temperatura foram determinados no momento da amostragem, in loco. Os demais parâmetros, CODT, DBO e DQO, foram determinados em laboratório.

Para a avaliação dos parâmetros de qualidade da água em termos qualitativos e, principalmente, em termos de dispersão de suas respectivas concentrações ao longo da seção transversal do rio, foram realizadas análises estatísticas segundo os pressupostos de Fonseca e Martins (1996) para classificar os coeficientes de variação em baixa dispersão (CV $\leq 10 \%)$, média dispersão $(10 \%<\mathrm{CV} \leq 20 \%)$ e alta dispersão (CV > 20\%). A partir da avaliação dos resultados de dispersão entre os pontos de monitoramento, será possível sugerir se os resultados qualitativos mensurados ao longo da seção são representativos da mesma (baixa dispersão) ou não (alta dispersão). Ou seja, se os valores determinados nas margens do rio são tão representativos da área em estudo quanto os valores determinados na seção central do rio, por exemplo. Adicionalmente, para os parâmetros de qualidade que apresentaram as maiores dispersões, ou o predomínio de alta dispersão durante o período avaliado, com auxílio do software Statistica $7.0 \AA$, foram realizadas as análises de variância, ANOVA, com 95\% de intervalo de confiança, Teste F e o valor $\mathrm{p}$, as quais indicam se a diferença entre os pontos sugerida pela análise de dispersão é significativa estatisticamente ou não. 


\section{RESULTADOS E DISCUSSÃO}

Os resultados obtidos por este trabalho são discutidos quanto à variação existente entre os pontos de monitoramento situados ao longo da seção transversal do rio segundo os parâmetros de condutividade, $\mathrm{pH}$, turbidez, temperatura, OD, COD, DBO e DQO.

Para verificar o grau de dispersão entre os pontos ao longo da seção transversal do rio em estudo, foram realizadas análises estatísticas distintas. A Tabela 1 apresenta para cada parâmetro monitorado seus respectivos resultados de média e desvio padrão, segundo coletas, lembrando que cada coleta aqui contém informações qualitativas referentes aos cinco pontos amostrais.

Tabela 1. Média e Desvio Padrão segundo parâmetros monitorados

\begin{tabular}{lcccccc}
\hline \multirow{2}{*}{ Parâmetro } & \multicolumn{6}{c}{ Coleta } \\
\cline { 2 - 7 } & $\mathrm{C} 1$ & $\mathrm{C} 2$ & $\mathrm{C} 3$ & $\mathrm{C} 4$ & $\mathrm{C} 5$ & $\mathrm{C} 6$ \\
\hline Condutividade $^{1}$ & $266,4 \pm 4,77$ & $203,2 \pm 20,28$ & $212,0 \pm 7,18$ & $239,6 \pm 7,4$ & $347,8 \pm 7,36$ & $238,0 \pm 7,78$ \\
$\mathrm{pH}$ & $7,19 \pm 0,03$ & $7,19 \pm 0,03$ & $7,27 \pm 0,06$ & $7,39 \pm 0,11$ & $7,03 \pm 0,11$ & $7,31 \pm 0,04$ \\
Turbidez & $36,16 \pm 4,14$ & $29,68 \pm 2,95$ & $41,0 \pm 2,46$ & $31,56 \pm 1,52$ & $19,52 \pm 0,73$ & $51,18 \pm 2,17$ \\
Temperatura $^{3}$ & $23,36 \pm 0,18$ & $22,22 \pm 0,13$ & $18,06 \pm 0,21$ & $17,98 \pm 0,65$ & $19,06 \pm 0,25$ & $18,42 \pm 0,37$ \\
OD $^{4}$ & $0,55 \pm 0,33$ & $1,81 \pm 0,08$ & $1,79 \pm 0,17$ & $2,62 \pm 0,1$ & $1,44 \pm 0,27$ & $1,63 \pm 0,24$ \\
CODT $^{4}$ & $7,29 \pm 0,59$ & $5,68 \pm 0,46$ & $2,89 \pm 0,13$ & $4,11 \pm 2,15$ & $3,32 \pm 0,95$ & $5,04 \pm 1,47$ \\
DBO $^{4}$ & $11,69 \pm 6,65$ & $2,76 \pm 0,51$ & $5,38 \pm 1,48$ & $6,28 \pm 1,18$ & $4,93 \pm 2,18$ & $5,89 \pm 1,42$ \\
DQO $^{4}$ & $94,72 \pm 20,44$ & $26,98 \pm 7,92$ & $28,53 \pm 9,9$ & $50,56 \pm 6,02$ & $15,12 \pm 1,44$ & $26,48 \pm 5,84$ \\
\hline
\end{tabular}

Nota: ${ }^{1} \mu \mathrm{Scm}^{-1} ;{ }^{2} \mathrm{NTU} ;{ }^{\circ} \mathrm{C} ;{ }^{4} \mathrm{mgL}^{-1}$

Vale salientar que durante a amostragem foram determinados os seguintes valores de vazão para cada coleta: $Q_{C 1}=50,88 \mathrm{~m}^{3} \mathrm{~s}^{-1} ; \mathrm{Q}_{\mathrm{C} 2}=102,44 \mathrm{~m}^{3} \mathrm{~s}^{-1} ; \mathrm{Q}_{\mathrm{C} 3}=97,1$ $\mathrm{m}^{3} \mathrm{~s}^{-1} ; \mathrm{Q}_{\mathrm{C} 4}=73,0 \mathrm{~m}^{3} \mathrm{~s}^{-1} ; \mathrm{Q}_{\mathrm{C} 5}=30,0 \mathrm{~m}^{3} \mathrm{~s}^{-1} ; \mathrm{e} \mathrm{Q}_{\mathrm{C} 6}=27,3 \mathrm{~m}^{3} \mathrm{~s}^{-1}$. A Figura 3 contém os resultados de coeficiente de variação $(\mathrm{CV})$, para os parâmetros em avaliação também segundo coletas. Conforme mencionado anteriormente, foram assumidas como baixa dispersão CV $\leq 10 \%$, media dispersão $10 \%<\mathrm{CV} \leq 20 \%$, e alta dispersão CV > 20\% (FONSECA; MARTINS, 1996). 


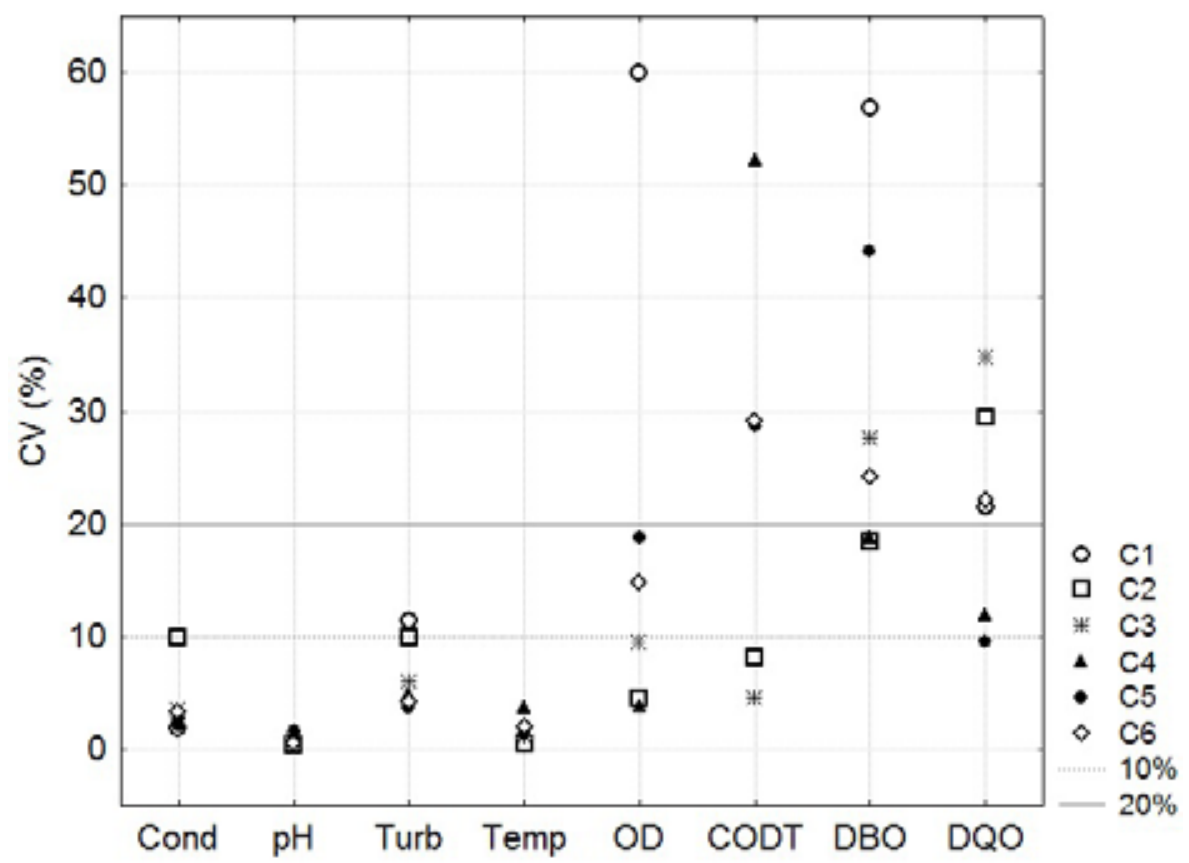

Figura 3. Coeficiente de Variação, CV segundo parâmetros monitorados.

De acordo com a Figura 3, que apresenta a dispersão com base no CV, juntamente com a Tabela 1 , que contém demais informações estatísticas, tornou-se possível realizar para cada coleta uma prévia avaliação quanto ao grau de dispersão entre os pontos situados ao longo da seção do rio, sendo que uma alta dispersão pode sugerir diferenças significativas entre tais pontos. Assim constatou-se que:

1) A condutividade apresentou baixa dispersão entre os pontos monitorados segundo uma mesma coleta, sendo que a maior dispersão observada ocorreu na coleta C2 (desvio padrão de 20,28 e CV de 9,98\%), porém, ainda categorizada como de baixa dispersão. Em termos de qualidade da água, os resultados obtidos durante o período monitorado foram elevados (média mínima de 203,2 $\mu \mathrm{cm}^{-1}$ e máxima de $347,8 \mu \mathrm{cm}^{-1}$ ), dado que segundo CETESB (2009), valores de condutividade superiores a $100 \mu \mathrm{cm}^{-1}$ caracterizam um ambiente impactado. 
2) $\mathrm{O} \mathrm{pH}$ apresentou baixa dispersão (quase nula) durante todo o período monitorado (desvio padrão máximo de 0,11 ). O valor de $\mathrm{pH}$ exerce influência sobre processos biológicos e químicos - ionização, volatilidade, dissolução, precipitação, toxicidade, entre outros (WEINER, 2000), e sua estabilidade neste caso pode estar relacionada, entre outras coisas, com o potencial de alcalinidade e acidez do meio aquático. $\mathrm{Na}$ análise qualitativa dos resultados, pode-se afirmar que durante o período monitorado, o parâmetro manteve-se em conformidade com classe 2 de enquadramento do manancial, como disposto na Resolução CONAMA n ${ }^{\circ}$ 357/2005 a qual estabelece um range ótimo de 6,0 $<\mathrm{pH}<9,0$ (BRASIL, 2005).

3) A turbidez apresentou baixa dispersão, com exceção da coleta C1 que apresentou coeficiente de variação de $11,45 \%$, o qual a classifica como de média dispersão, e desvio padrão de 4,14. Em termos de enquadramento, a turbidez manteve-se em conformidade com a classe 2 que de acordo com a Resolução CONAMA no 357/2005 (BRASIL, 2005) apresenta um limite máximo de até 100 NTU.

4) Os resultados de temperatura apresentaram baixa dispersão, e qualitativamente compreenderam faixas consideradas aceitáveis para a manutenção da fauna e flora aquáticas (HUCKEMBECK et al., 2012). Comumente corpos d'água apresentam variações de temperatura ao longo do dia e das estações do ano. Entretanto, o lançamento de efluente com temperatura elevada pode impactar ambientes aquáticos por interferir em parâmetros físicos como de tensão superficial e viscosidade, além de alterar taxas de reações químicas e biológicas, e o desenvolvimento de determinadas espécies.

5) Para o OD, a análise dos resultados expressos na Figura 3 e na Tabela 1 permite afirmar uma (i) baixa dispersão encontrada durante as coletas C2, C3, e C4; (ii) média dispersão durante as coletas C5 e C6; e (iii) alta dispersão na coleta $\mathrm{C} 1$ (média de $0,55 \mathrm{mgL}^{-1}$, desvio padrão de $0,33 \mathrm{e} \mathrm{CV}$ de 60\%). Contrariamente à estabilidade química sugerida pelos parâmetros de condutividade, $\mathrm{pH}$, turbidez e temperatura, constatou-se, a partir dos resultados de oxigênio dissolvido obtidos, uma elevada variação de 
concentração do parâmetro ao longo da seção. Quanto à análise de qualidade do corpo d'água, as concentrações de oxigênio dissolvido mensuradas estão a nível considerado deficitário e em desconformidade com a classe, segundo padrões estabelecidos pela Resolução CONAMA n ${ }^{0}$ 357/2005 (BRASIL, 2005) que exige um mínimo de $5 \mathrm{mgL}^{-1}$ no enquadramento em classe 2. Os valores de oxigênio dissolvido inferiores a $4 \mathrm{mgL}^{-1}$ estão relacionados a um ambiente severamente poluído (WEINER, 2000). O déficit de oxigênio dissolvido sugere a presença de compostos passíveis de oxidação, dado que o ponto de estudo está situado à jusante das sub-bacias dos rios Palmital, Belém e Barigui, as quais são altamente urbanizadas (KNAPIK, 2009). Logo, seria coerente supor como sumidouros de oxigênio, a presença de matéria orgânica lábil e de compostos nitrogenados possivelmente advindos de despejos domésticos e industriais.

6) Os resultados de dispersão amostral referentes ao CODT apresentaram variações consideradas de alta dispersão durante as coletas $\mathrm{C} 4, \mathrm{C} 5$ e C6, e baixa dispersão para as demais. De modo geral, os resultados médios por coleta para o CODT foram inferiores (com exceção de C2) aos respectivos de DBO, fato que convém salientar que as análises de DBO compreendem a mensuração da fração total biodegradável (dissolvida e particulada) da amostra, e já o CODT consiste na mensuração de todo o carbono presente na fração dissolvida da amostra (GONÇALVES, et al., 2011). Logo, sugere-se que durante a coleta $\mathrm{C} 2$ a fração de matéria orgânica biodegradável estava menos biodisponível aos microrganismos decompositores.

7) Quanto a DBO, as coletas apresentaram classificação variada em alta dispersão (C1, C3, C5 e C6) e média dispersão (C2 e C4). Em termos de qualidade, verificou-se certo grau de dificuldade em avaliar os resultados de DBO quanto ao respeito à classe 2 , pois na análise isolada dos resultados médios de DBO por coleta constatou-se que apenas as coletas C2 e C5 estavam em conformidade legal. Contudo, devido ao predomínio de elevados valores de desvio padrão, confirmados pela alta dispersão dos dados, salienta-se que a conformidade ou não à classe de qualidade pode gerar questionamentos. 
(conclusão)

8) Já para DQO, foi constatada alta dispersão durante as coletas C1, C2, C3 e C6; média dispersão durante a coleta $\mathrm{C} 4$; e baixa dispersão durante a coleta C5. Destaque para a coleta C3 em que a média foi de $28,53 \mathrm{mgL}^{-1}$, o desvio padrão assumiu valores de 9,9 e o CV foi de $35 \%$.

A estabilidade de parâmetros de qualidade da água ao longo da seção transversal (neste caso, os parâmetros de condutividade, $\mathrm{pH}$, turbidez e temperatura) indicam de certa forma o grau de equilíbrio do sistema aquático, especialmente o de temperatura que exerce influência direta na velocidade e no grau de inúmeras reações químicas, físicas e biológicas. Contudo, este equilíbrio alcançado não tem relação com um ambiente de qualidade, pelo contrário, neste caso caracteriza um sistema sob uma condição indesejada, ou poluída a julgar pelos resultados qualitativos de condutividade, turbidez, OD, COD, DBO e DQO.

De modo geral, a análise dos resultados realizada a partir da Tabela $1 \mathrm{e}$ da Figura 3 permite afirmar que os parâmetros de maior variação ao longo da seção transversal em estudo foram os de caráter orgânico, neste caso: COD, DBO e DQO, e o oxigênio dissolvido que possui relação estreita com os parâmetros orgânicos, diante dos processos metabólicos de degradação aeróbia da matéria orgânica que alteram a concentração de OD no ambiente aquático.

Constata-se que casos como estes podem promover dificuldades de interpretação dos dados, e reduzir a credibilidade dos mesmos. Variações extremas de concentração ao longo de uma seção de estudo prejudicam ou mesmo inviabilizam as atividades de monitoramento. Para efeito ilustrativo da variabilidade espacial e temporal dos resultados, a Figura 4 e a Figura 5 apresentam respectivamente as trajetórias espaço-temporal de dois parâmetros orgânicos de maior dispersão observada, Demanda Bioquímica de Oxigênio - DBO e Demanda Química de Oxigênio - DQO. 


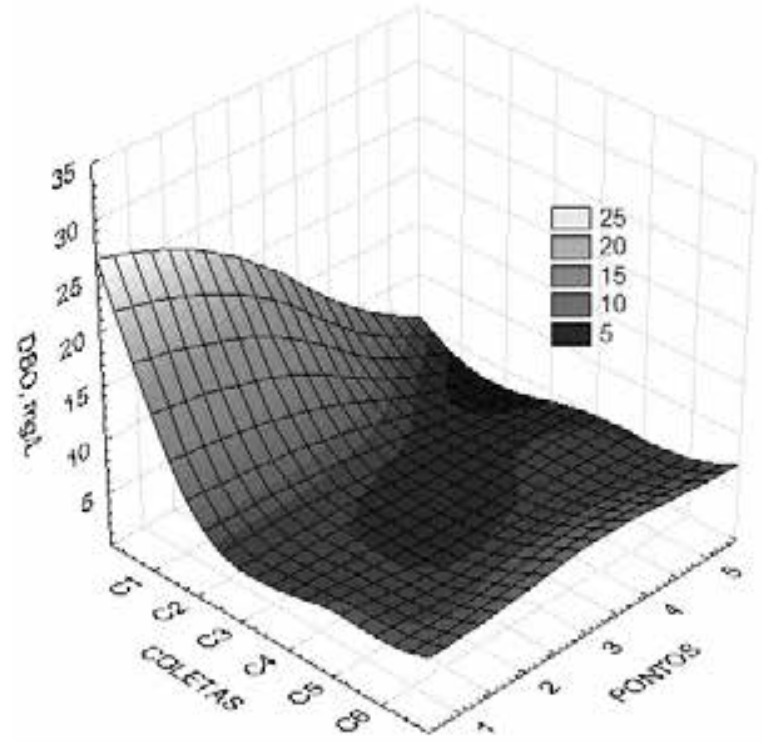

Figura 4. Dispersão espaço-temporal referente ao monitoramento da DBO.

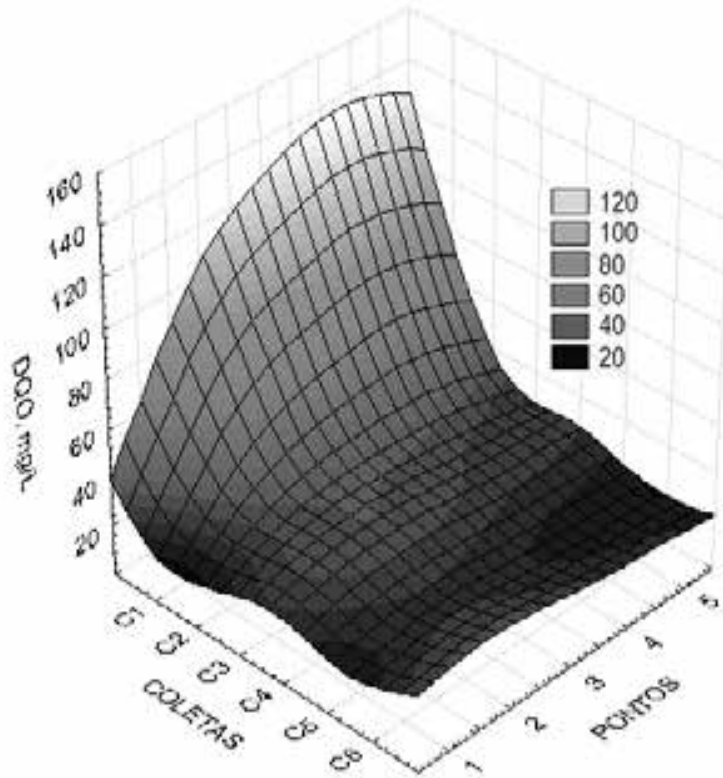

Figura 5. Dispersão espaço-temporal referente ao monitoramento da DQO. 
A avaliação espaço-temporal permite afirmar em primeira análise que os resultados referentes aos dois parâmetros em questão foram amortizados pela primeira coleta, $\mathrm{C} 1$. Se comparada com as demais coletas, pode-se observar que a coleta $\mathrm{C} 1$ apresentou elevados valores médios de carga orgânica, sendo de $11,69 \mathrm{mgL}$ ${ }^{1}$ de DBO e 94,72 mgL ${ }^{-1}$ de DQO. Trata-se de uma condição de elevada degradação do ambiente aquático. Na análise particular dos resultados de DBO, observa-se um leve gradiente de maior concentração no ponto 1, P1 (margem direita) para uma menor concentração no ponto 5, P5 (margem esquerda). Neste contexto, os resultados de DQO apresentaram uma tendência de gradiente de maior concentração nos pontos centrais, $\mathrm{P} 2$, P3 e P4 e margem esquerda (especialmente em C1), e de menor concentração na margem direita. Os pontos situados nas margens do rio estão sob condições geomorfológicas de fundo, e consequentemente de fluxo, diferenciadas, a julgar pela Figura 2 que apresenta a configuração do leito da seção de estudo. De modo geral, esta é a configuração típica de rios, o que torna usual que o fluxo de velocidades nas margens seja menor que o fluxo nas regiões centrais do corpo hídrico. A possibilidade de regiões de remanso ou "zonas mortas" nos ambientes de margem justificam as sugestões dispostas na NBR no 9.897 (ABNT, 1987a) quanto ao número de pontos amostrais ao longo de uma seção transversal do rio em estudo. Ou seja, parte-se do pressuposto que as concentrações obtidas a partir da margem possam reproduzir impacto no monitoramento do corpo hídrico pela possibilidade de não representarem a realidade de toda a seção transversal do rio, ou em outras palavras, por possivelmente promover resultados diferentes dos praticados no fluxo central do rio.

Contudo, quanto maior o número de pontos amostrais, maior será a complexidade e o custo para a prática de monitoramento conforme a normativa, especialmente no caso do monitoramento da qualidade da água de grandes bacias hidrográficas. Entretanto, é de conhecimento geral que os corpos hídricos apresentam características peculiares de, por exemplo, velocidade de fluxo, geomorfologia do leito de fundo, vazão, capacidade de autodepuração, etc. Sendo que este fato também é verdadeiro para as "n" seções transversais do rio, ou seja, cada seção é única. E se esta "seção única" apresenta um comportamento de dinâmica de fluxo característico em que as concentrações na margem sejam menores quando comparadas com as de 
centro, isso não significa que esta variação seja significativa. Logo, pouco se sabe da real necessidade em adotar o sugerido pela norma mencionada, dado que não há o consenso em realizar uma investigação sobre a significância da diferença existente entre os pontos amostrais ao longo da seção transversal do rio.

Neste contexto, vale reforçar que não foram encontrados estudos com o enfoque aqui abordado, de avaliação da dispersão da concentração dos parâmetros ao longo de uma seção. Assim, dada à alta dispersão encontrada ao longo do período monitorado para determinados parâmetros (Figura 3), e para atestar se os pontos monitorados apresentavam ou não uma diferença significativa foi realizada uma Análise de Variância - ANOVA segundo os parâmetros de OD, COTD, DBO e DQO (Figura 6). Para a referida análise, os cinco pontos ao longo da seção transversal foram avaliados entre si quanto a possíveis diferenças significativas. A ANOVA realizada entre os pontos parte do princípio que cada ponto (tratamento) estava sob as mesmas condições ambientais em uma mesma coleta (repetições).

Além da Análise de Variância com intervalo de confiança de $95 \%$ foram calculados os seguintes valores de Teste $\mathrm{F}$ e valor $\mathrm{p}$ para os parâmetros de qualidade avaliados: OD com $\mathrm{F}=0,13280$ e $\mathrm{p}=0,96885$; CODT com $\mathrm{F}=0,22691$ e $\mathrm{p}=$ 0,92074; DBO com $\mathrm{F}=0,38404$ e $\mathrm{p}=0,81793$; e DQO com $\mathrm{F}=0,13596$ e $\mathrm{p}=$ 0,96750 . Para o intervalo de confiança adotado tem-se o valor tabelado do Teste $\mathrm{F}$ de $\mathrm{F}(4,25)=2,76$ (maior que os respectivos calculados), o que consequentemente torna a hipótese nula $\mathrm{H}_{0}$ verdadeira, ou seja, os pontos não diferem significativamente. Fato que é confirmado pelos respectivos resultados de valor $\mathrm{p}$, o qual indica o limite de significância para aceitar $\mathrm{H}_{0}$ e pode ser visualizado graficamente por meio da Figura 6. 

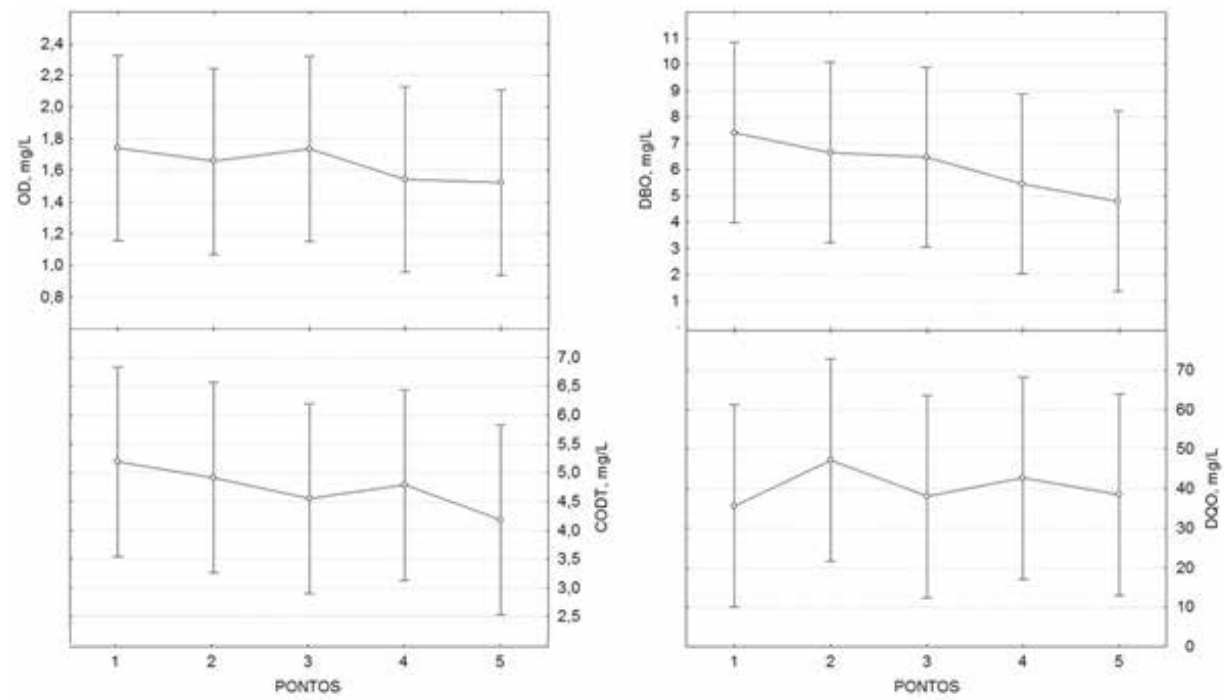

Figura 6. ANOVA entre os pontos de monitoramento para os parâmetros de OD, CODT, DBO, DQO.

Em termos de monitoramento de recursos hídricos, a Análise de Variância permite afirmar, para este estudo de caso, que o local da amostragem ao longo da seção transversal do rio não interfere no resultado final, uma vez que os cinco pontos amostrados são estatisticamente iguais e representativos dos fenômenos que ocorrem na seção do rio. Logo, a alta dispersão encontrada entre os pontos, especialmente quanto aos parâmetros de OD, DBO, DQO e CODT (Figura 3), não foi estatisticamente significativa para predizer que as margens do rio possuem características qualitativas diferentes da região central do mesmo rio, na mesma seção transversal, conforme as análises de ANOVA, Teste $\mathrm{F}$ e p-valor. Logo, o monitoramento da quali-quantitativo dos cinco pontos distribuídos ao longo da seção de rio em estudo, pode ser simplificado com a adoção de uma amostragem em um único ponto, independente de sua localização ao longo da seção.

Este trabalho é pioneiro e apresenta resultados importantes de auxílio na tomada de decisão de assuntos relacionados e gestão e monitoramento de recursos hídricos, pois pode simplificar as atividades do monitoramento por meio da redução do número de pontos amostrais sugeridos pela NBR n ${ }^{0} 9897$ (ABNT, 1978a), o que, consequentemente, reduzirá os custos e tempo amostral, laboratorial 
e de processamento de dados. Enfim, tratam-se de resultados preliminares, mas que podem auxiliar ou mesmo se tornar uma prática comum no planejamento amostral de corpos hídricos quanto a definição do real número de pontos amostrais a serem monitorados.

\section{CONSIDERAÇÕES FINAIS}

Com o objetivo de investigar a dispersão da concentração de certos parâmetros de qualidade ao longo da seção transversal do rio em estudo, foram realizadas distintas avaliações como grau de dispersão, análise espaço-temporal e Análise de Variância, ANOVA. A partir da análise de dispersão dos pontos ao longo da seção foi possível concluir o predomínio de baixa dispersão durante a mensuração dos parâmetros de condutividade, $\mathrm{pH}$, turbidez e temperatura; e o predomínio de média a alta dispersão na estimação dos parâmetros de oxigênio dissolvido, carbono orgânico dissolvido, demanda bioquímica de oxigênio e demanda química de oxigênio. Na avaliação espaço-temporal realizada para os parâmetros de maior dispersão, DBO e DQO, foi possível identificar uma leve tendência de distribuição da concentração ao longo da seção transversal do rio, em que, de maneira geral, os pontos amostrais situados nas margens apresentaram concentrações orgânicas menores quando comparados com os pontos centrais. Com o propósito de mensurar o possível grau de impacto ao sistema de gestão de recursos hídricos decorrentes de dúbia análise e interpretação das informações coletadas durante o monitoramento, foi realizada a Análise de Variância para os parâmetros enquadrados em média a alta dispersão, de modo a atestar a real dispersão existente entre os pontos amostrais. A partir da ANOVA foi possível afirmar para este estudo de caso que os cinco pontos ao longo da seção são significativamente iguais. Isso sugere que a localização do ponto de amostragem ao longo da seção do rio pode não refletir em erros passíveis de causar impacto de interpretação e de representação dos resultados. Adicionalmente, para este caso, adotar uma distribuição de vários pontos amostrais ao longo de uma seção para representá-la, conforme sugerido pela NBR no 9.897 (ABNT, 1987a), torna-se desnecessário. 
Em termos de qualidade da água, os valores obtidos de $\mathrm{pH}$, turbidez e temperatura enquadraram-se em conformidade com o estabelecido pela resolução CONAMA $n^{0} 357 / 2005$ (BRASIL, 2005). Porém, nos demais parâmetros houve o predomínio de valores característicos de um ambiente impactado negativamente.

Por fim, este estudo agrega uma experiência importante para a prática de planejamento da gestão de recursos hídricos. Trata-se de uma investigação pontual, com resultados conclusivos sobre uma técnica de amostragem que pode ser otimizada sem promover impacto no resultado final. Tal sistema de investigação pode ser adotado como procedimento padrão durante o planejamento do monitoramento de corpos d'água de modo a minimizar sua complexidade ao reduzir o número de pontos amostrais (se não constatada variação significativa entre tais), sem comprometer a confiabilidade dos resultados.

\section{AGRADECIMENTOS}

Esta pesquisa foi financiada com recursos da CAPES-REUNI e do CNPq.

\section{REFERÊNCIAS}

ABNT - Associação Brasileira de Normas Técnicas. NBR 9.897: Planejamento de Amostragem de Efluentes Líquidos e Corpos Receptores. Rio de Janeiro: ABNT, $1987 \mathrm{a}$.

ABNT - Associação Brasileira de Normas Técnicas. NBR 9.898: Preservação e Técnicas de Amostragem de Efluentes Líquidos e Corpos Receptores. Rio de Janeiro: ABNT, $1987 \mathrm{~b}$.

ABNT - Associação Brasileira de Normas Técnicas. NBR 12614: Águas - Determinação da demanda bioquímica de oxigênio (DBO) - Método de incubação $\left(20^{\circ} \mathrm{C}\right.$, cinco dias). Rio de Janeiro: ABNT, 1992.

APHA. Standard Methods for the Examination of Water and Wastewater. 20th ed. 
Washington, DC: APHA, 1998.

BARBOT, E.; VIDIC, N. S.; GREGORY, K. B.; VIDIC, R. D. Spatial and Temporal correlation of water quality parameters of produced waters from devonian-age shale following hydraulic fracturing. Environ. Sci. Technol. v. 6, n. 47, p. 2562-2569, 20 fev. 2013.

CAMPANHA, M. B.; MELO, C. A.; MOREIRA, A. B.; FERRARESE, R. F. M. S.; TADINI, A. M.; GARBIN, E. V.; BISINOTI, M. C. Variabilidade espacial e temporal de parâmetros físico-químicos nos rios Turvo, Preto e Grande no Estado de São Paulo, Brasil. Química Nova, São Paulo, v. 33, n. 9, p. 1831-1836, 2010.

CETESB. Variáveis de qualidade das águas. Disponível em: < http://www.cetesb. org.br > . Acesso em: 26 ago. 2014.

COELHO, M.; KISHI, R. T. Caracterização de Cargas Orgânicas nos Rios Barigui, Belém e Atuba na Bacia Hidrográfica do Alto Iguaçu. In: SIMPÓSIO BRASILEIRO DE RECURSOS HÍDRICOS, 19., 2011, Maceió. Anais... Maceió: Associação Brasileira de Recursos Hídricos, 2011.

BRASIL. CONAMA. Resolução $\mathrm{n}^{\mathrm{o}} 357$ de 17 de março de 2005. Dispõe sobre a classificação e diretrizes ambientais para o enquadramento dos corpos de água superficiais, bem como estabelece as condições e padrões de lançamento de efluentes. Relator: Marina Silva. Diário Oficial da União, Brasília, 18 de março de 2005.

PARANÁ. Superintendência dos Recursos Hídricos e Meio Ambiente - SUREHMA. Portaria SUREHMA no 20 de 12 de maio de 1992. Bacia do Rio Iguaçu. Diário Oficial do Estado, Curitiba, 20 maio 1992.

FRANÇA, M. S. Análise estatística multivariada dos dados de monitoramento de qualidade de água da Bacia do Alto Iguaçu: uma ferramenta para gestão de recursos hídricos. Curitiba, 2009. 150f. Dissertação (Mestrado) - Universidade Federal do Paraná, Setor de Tecnologia, Curitiba, 2009. 
FONSECA, J. S.; MARTINS, G. A. Curso de estatística. 6. ed. São Paulo: Atlas, 1996.

GONÇALVES, V. D. Avaliação de alterações de parâmetros de qualidade da água em uma seção transversal: seu impacto para a gestão de recursos hídricos. Curitiba, 2011. Dissertação (Mestrado) - Universidade Federal do Paraná, 2011.

GONÇALVES, V. D.; FERNANDES, C. V. S.; BEM, C. C.; KNAPIK, H. G.; MANNICH, M. Desafios da Gestão de Recursos Hídricos: um olhar sobre o monitoramento ambiental de rios. In: CONGRESSO BRASILEIRO DE ENGENHARIA SANITÁRIA E AMBIENTAL, 26. Anais... Porto Alegre, 2011b.

HUCKEMBECK, S.; CLAUDINO, M.; CORREA, F.; BASTOS, R. F.; LOEBMANN, D.; TOZETTI, A. M.; GARCIA, A. M. The activity patterns and microhabitat use of Pseudis minuta Günther, 1858 (Anura, Hylidae) in the Lagoa do Peixe National Park, a biosphere reserve of the brazilian subtropics. Braz. J. Biol., v. 72, n. 2, p. 331-336, 31 mai. 2012.

KAMIYAMA, H. A complexidade do DBO. DAE. v. 48, n. 152, set. 1988.

KNAPIK, H. G. Reflexões sobre monitoramento, modelagem e calibração na gestão de recursos hídricos: estudo de caso da qualidade da água da bacia de recursos hídricos. Curitiba, 2009. 180f. Dissertação (Mestrado) - Universidade Federal do Paraná, Setor de Tecnologia, Curitiba, 2009.

LIU, Y.; ZHENG, B.; WANG, M.; XU, Y.; QIN, Y. Optimization of sampling frequency for routine river water quality monitoring. Science China Chemistry. v. 57, n. 5, Mai. 2014.

MEMARZADEH, M.; MAHJOURI, N.; KERACHIAN, R. Evaluating sampling locations in river water quality monitoring networks: application of dynamic factor analysis and discrete entropy theory. Environ Earth Sci. v. 70, p. 2577-2585, Fev. 2013.

PORTO, M. F. et al. Bacias críticas: bases técnicas para a definição de metas progressivas para seu enquadramento e a integração com os demais instrumentos de gestão. Curitiba: UFPR - FINEP/CT-HIDRO, 2007. 
REIS, A.; PARKER, A.; ALENCOÃO, A. Avaliação da qualidade de sedimentos em rios de montanha: um caso de estudo no norte de Portugal. Recursos Hídricos Associação portuguesa dos Recursos Hídricos. v. 31, 2001.

SANDERS, T. G.; WARD, R. C.; LOFTIS, J. C.; STEELE, T. D.; ADRIAN, D. D.; YEVJEVICH, V. Design of networks for monitoring water quality. Water Resources Publications LLC, account for pollution load along with number of tributaries. Highlands Ranch, Colorado, USA, 1983.

SILVA, J. P. A.; MUELBERT, A. E.; OLIVEIRA, E. C.; FÁVARO, L. F. Reproductive tactics used by the Lambari Astyanax aff. fasciatus in three water supply reservoirs in the same geographic region of the upper Iguaçu River. Neotropical Ichthyology, v. 8, n. 4 , p. 885-892, 2010.

STROBL, R. O.; ROBILLARD, P. D. Network design for water quality monitoring of surface freshwaters: A review. Journal of Environmental Management. v. 87, p. 639-648, 2008.

WEINER, E. R. Applications of environmental aquatic chemistry: a practical guide. 2. ed. Boca Raton, FL: CRC Press, 2000.

Recebido em: 18 de setembro de 2015 Aceito em: 23 de abril de 2016 